\title{
A review on the artificial neural network applications for small-signal modeling of microwave FETs
}

\author{
Zlatica Marinković $^{1}$ (i) | Giovanni Crupi ${ }^{2}$ (1) | Alina Caddemi ${ }^{3}$ | Vera Marković $^{1}$ | \\ Dominique M.M.-P. Schreurs ${ }^{4}$
}

\footnotetext{
${ }^{1}$ Faculty of Electronic Engineering, University of Niš, Niš, Serbia

${ }^{2}$ BIOMORF Department, University of Messina, Messina, Italy

${ }^{3}$ Department of Engineering, University of Messina, Messina, Italy

${ }^{4}$ Electronic Engineering Department, KU Leuven, Leuven, Belgium
}

\section{Correspondence}

Zlatica Marinković, Faculty of

Engineering, University of Niš, Niš 18000 , Serbia.

Email: zlatica.marinkovic@elfak.ni.ac.rs

\section{Funding information}

Serbian Ministry for Education and Science, Grant/Award Number: TR-32052

\begin{abstract}
The purpose of this paper is to provide a comprehensive overview of the fieldeffect transistor (FET) small-signal modeling using artificial neural networks (ANNs). To gain an in-depth insight into how to effectively develop an ANN model, we present a comparative study on the application of the ANNs for modeling the scattering $(S$-) parameters of a variety of FET technologies versus bias point, ambient temperature, and geometrical dimensions. As will be shown, the main challenge consists of identifying the most appropriate ANN model for the specific case under study. This is because the performance of an ANN-based model can vary significantly, depending especially on the choice of the model structure and the size and parameters of the chosen ANN. In addition, the choice of the model is related directly to the behavior of the FET characteristics, which might greatly depend on the selected device technology and operating conditions. The analysis of the present comparative study allows understanding how to properly construct ANN models to perform at their best for a successful FET modeling.
\end{abstract}

\section{KEYWORDS}

artificial neural networks, FET, microwave measurements, semiconductor device modeling

\section{1 | INTRODUCTION}

Artificial neural networks (ANNs) are a well-established and very powerful mathematical tool, finding a variety of applications as a modeling tool in the field of RF and microwaves. ${ }^{1-39}$ One of the most attractive features of ANNs is their ability to learn and generalize from a set of training data, which is suitable to be exploited for building device models from the measured characteristics. Therefore, ANNs are widely used also for modeling and predicting the scattering $\left(S\right.$-) parameters of microwave field-effect transistors (FETs).$^{7,8,11,13,18,40-49}$ The main advantage of an ANN-based FET model is that, because of the "black-box" nature of ANN models, the $S$-parameters can be straightforwardly and accurately reproduced without requiring the extraction of an equivalent-circuit model ${ }^{50-68}$ or a detailed knowledge of the FET physics. ${ }^{69-73}$ As a matter of fact, by exploiting ANNs, it is possible mathematically describe the observable inputoutput relationships. Nevertheless, it can be quite challenging to determine the most appropriate ANN model for the specific case study, depending on the FET technology and operating conditions. Within this context, we present a comparative study on the basis of analyzing experimental results carried out on many FET devices working under different 
conditions, showing how to build ANNs for modeling the peculiarities of each particular case. The investigated FETs are based on different semiconductor technologies: gallium arsenide (GaAs), gallium nitride ( $\mathrm{GaN})$, and silicon ( $\mathrm{Si}$ ).

In all cases, the ANNs have frequency and operating conditions (eg, bias point, temperature, and device dimensions) as inputs and the $S$-parameters as outputs. Depending on device technology and the range of the considered operating conditions, the number of the ANNs composing the model differs, in order to model and predict the behavior of the analyzed $S$-parameters. Moreover, in certain cases, it is necessary to introduce more complex structures of neural models.

In the case of GaAs high electron mobility transistor (HEMTs), an ANN model covering a wide range of considered biases is developed for each of the $S$-parameters. A single ANN modeling, both the real and imaginary parts, is used for each of the two reflection coefficients $S_{11}$ and $S_{22}$, while two ANNs modeling, separately the real and imaginary parts, are required for each of the two transmission coefficients $S_{21}$ and $S_{12}$, because of their more complicated behavior. ${ }^{42,43}$ Nevertheless, if the model is developed for a narrower bias range, such as for the bias conditions for typical applications of the device, a single ANN can be enough for modeling all of the $S$-parameters. ${ }^{40,41}$

The GaN HEMTs are well suited for high-power high-frequency applications, and typically, they have a relatively high magnitude of $S_{21}$. A practical consequence of this peculiarity might be that the logarithmic representation can be necessary for both $S_{21}$ magnitude and frequency, in order to make dynamics of the $S_{21}$ magnitude smaller and thus to improve ANN performance. ${ }^{47,48}$

In case of Si FinFETs, the four $S$-parameters might be affected by low-frequency kinks, because of the lossy Si substrate. The losses in the substrate can be taken into account by using the so-called prior knowledge input (PKI) neural approach, consisting of developing a first an ANN model for reproducing the real parts of the $S$-parameters at low frequencies and to use outputs of this model as the prior knowledge for the second ANN model reproducing the $S$-parameters over the full frequency range. It should be noticed that the PKI neural approach is not required for modeling the $S$ parameters obtained after applying the two-step de-embedding procedure based on "open" and "short" dummy structures, as this procedure can enable removing the low-frequency kinks. ${ }^{44}$

The Si FinFET varactors, behaving as symmetric and reciprocal networks, require modeling of only two $S$-parameters: the input reflection parameter $S_{11}$ and the forward transmission parameter $S_{21}$. Moreover, after applying the deembedding procedure based on open dummy structure, the actual device behaves like a simple series impedance between anode and cathode, so it is enough to model $S_{11}$, and the three other parameters can be derived from it. However, although $S_{22}$ shows a behavior very close to $S_{11}$, the small differences between them can lead to very high percentage error for $S_{22}$ when its magnitude gets close to 0 , and therefore, it is recommended to develop a separate neural model for $S_{22 .}{ }^{46}$

In the case of Si metal-oxide-semiconductor field effect transistor (MOSFETs), a bias-dependent neural model scalable with the gate length is developed, as the effect of the gate-length scaling on the high-frequency MOSFET behaviors is of significant interest. The model for each of the $S$-parameters consists of two ANNs aimed at modeling the real and imaginary part of the relevant parameter. ${ }^{49}$

The rest of this paper is organized as follows. At the beginning, in section 2, the basic principles on the development of the considered neural models are given. In section 3, we report and discuss the experimental results. This section is organized into five subsections. For each subsection, a different device technology is analyzed: GaAs HEMTs, GaN HEMTs, Si FinFETs, Si FinFET varactors, and Si MOSFETs. Finally, we report the conclusive remarks in section 4.

\section{2 | NEURAL MODELS OF SMALL-SIGNAL S-PARAMETERS}

As mentioned in Introduction, a neural model of small-signal $S$-parameters consists of one or more ANNs having the $S$ parameters as outputs (see Figure 1). The number of input neurons (ie, size of the input layer) of each ANN corresponds

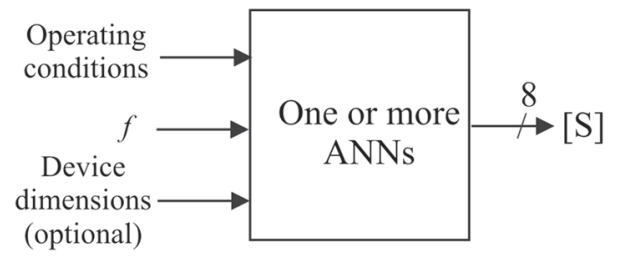

FIGURE 1 General ANN-based model of microwave transistor $S$-parameters 
to the number of input parameters. The number of output neurons (ie, size of the output layer) depends on the number of parameters being modeled by a particular ANN. The number of hidden neurons is determined during the model development. The input layer neurons have the unitary transfer functions (ie, they are used as a buffer); hidden layers have a sigmoid transfer function, and the output neurons have the linear transfer function.

In all of the presented cases, the ANN-training data sets consist of the measured $S$-parameters in the considered ranges of the model input parameter space. The size of the training and test data sets depend mainly on the available experimental data. In the presented work, the test sets were smaller than the corresponding training sets, except in the certain cases when a large number of measured data were available. When selecting the samples for training and test sets, it is very important to cover properly the input space and to include especially characteristic samples (such as samples referring to the input values that are on the edge of the considered input range or referring to the inputs where the $S$-parameters exhibit a particular behavior). In the case of significant number of samples available, one can try with different training set distributions. In such cases, we started first with a uniform distribution of the samples in the input space. If the models exhibited higher error and additional data were available in the area of input space with higher errors, as a first attempt to decrease the errors, more data referring to that input area were included in the training set. However, in some cases a nonuniform distribution did not help, since the errors were not mainly originated from the lack of training data. Therefore, it was necessary to develop a new model structure and/or to apply a certain additional processing of the input/output data (eg, a logarithmic representation instead of a linear representation), as it will be described in details in the next section. It should be mentioned that the training and test data were preprocessed so that all the input and output parameters were transferred in the $(-1,1)$ range in order to put the neurons in the most sensitive area of their transfer characteristics. This assumes that, after getting the ANN response, a postprocessing was to be done for obtaining the modeled parameters in the original range.

The number of hidden layers and their size (ie, number of neurons in each hidden layer) of an ANN cannot be a priori set, and therefore, they were determined during the training. Although in theory, one hidden layer can be enough to develop an accurate model, the experience has showed that one hidden layer was not enough to develop models when working with the transistor $S$-parameters versus biases and frequency. Therefore, all the models presented in this paper have two hidden layers. In the remaining of the text, the ANNs are denoted as $\mathrm{M}-\mathrm{H} 1-\mathrm{H} 2-\mathrm{N}$, where $\mathrm{M}, \mathrm{H} 1, \mathrm{H} 2$, and $\mathrm{N}$ are the numbers of the neurons in the input layer, in the first and the second hidden layers, and in the output layer, respectively.

To determine the number of hidden neurons for each ANN, a number of ANNs with different number of hidden neurons were trained, and the ANN giving the best modeling accuracy was chosen as the ANN for the final model. During the ANN validation test, both the learning capability (accuracy of the training data modeling) and generalization capability (accuracy of the modeling of data different form the training data) of the developed ANNs were tested and compared with the aim of choosing the ANN providing the best performance. In the training and test phase, the average test errors, the worst case errors, and the correlation coefficients, on the whole training and test sets, were calculated and compared. For most of the models, the final error estimation was based on calculating the percentage errors per a bias point averaged in the frequency range, as well as the maximum percentage errors per a bias point. However, in this paper, for the sake of space, information about the achieved errors is given only for the most representative cases, as the details about the validation and errors have been already published and the accuracy of the models have been already proven. The main focus here is on the differences in model structure depending on the technology. The term "model structure" refers primarily to the number of the ANNs needed to model all the four $S$-parameters and to the input and output parameters of the ANNs but not to the number of hidden layers and hidden neurons. Satisfactory accuracy on the training set prevented underlearning (under-fitting). To check if the ANNs exhibit over-learning (over-fitting), their response was examined with a smaller step than the step of sampling the inputs in the training set and preferably compared with the corresponding experimental data. However, in the case that the corresponding experimental data were not available, a number of visual inspections were done to spot the presence of an unexpected behavior in the $S$-parameters caused by the ANN over-fitting.

The expressions describing the developed ANN models, together with the expressions aimed at the input preprocessing and the output postprocessing, were implemented in a circuit simulator through variable and expression (VAR) blocks that have the same inputs as the inputs of the developed models and whose outputs corresponded to the $S$ parameters. The modeled transistor is represented as a two-port expression-defined network, which can be further used as a library element in the simulator. 


\section{3 | EXPERIMENTAL RESULS AND DISCUSSION}

The results given in this section refer to the following different device technologies: GaAs HEMTs, GaN HEMTs, Si FinFETs, Si FinFET varactors, and Si MOSFETs. For each of the technologies, only the most illustrative results are given. For a more extensive treatment of the subject, the reader is referred to our previous papers where we have described the developed models in details.

\section{1 | GaAs HEMTs}

The model shown in Marinković et $\mathrm{al}^{42}$ was developed for three AlGaAs/GaAs HEMTs with the same gate length $\left(L_{\mathrm{g}}=0.25 \mu \mathrm{m}\right)$ and differing in the gate width $(W=2 \times 50 \mu \mathrm{m}, 2 \times 100 \mu \mathrm{m}$, and $2 \times 150 \mu \mathrm{m})$. It was found that for achieving the satisfactory accuracy for $S_{11}$ and $S_{22}$, it was enough to develop a single ANN for modeling simultaneously the real and imaginary parts of each reflection coefficient (see Figure 2A). On the other hand, in the case of $S_{21}$ and $S_{12}$, it was necessary to model separately the real and imaginary parts of each transmission coefficient (ie, to use two ANNs having the structure shown in Figure 2B).

The model was developed for the frequency range from 0.5 to $50 \mathrm{GHz}$ and under the following bias range: $V_{\mathrm{ds}}$ from 0 to $2.5 \mathrm{~V}$ and $V_{\mathrm{gs}}$ from -1.5 to $0 \mathrm{~V}$. The data used for training were taken at 42 bias points, whereas the data used for model validation were taken at 34 bias points different from the ones used for training. The two output ANNs in Figure 2B have the structure 4-25-25-2, whereas the single-output ANNs in Figure 2A have the structure 4-25-25-1, except the ANN modeling the imaginary part of $S_{12}$ having the structure 4-21-20-1. For illustration, Figure 3 reports the comparison between measured and modeled $S$-parameters at a bias point not used for the model development: $V_{\mathrm{ds}}=2.5 \mathrm{~V}$ and $V_{\mathrm{gs}}=-0.6 \mathrm{~V}$. To make the plots clearer, $S_{21}$ is divided by 10 and $S_{12}$ multiplied by 10 . The percentage error averaged for all the four $S$-parameters at this bias point is less than $5 \%$ for all three considered devices. In general,

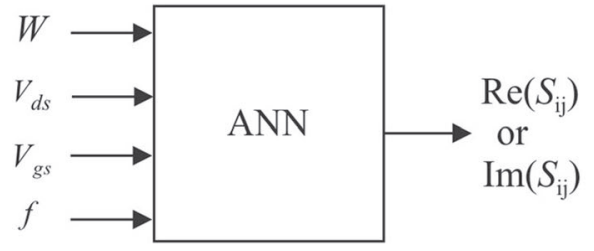

(A)

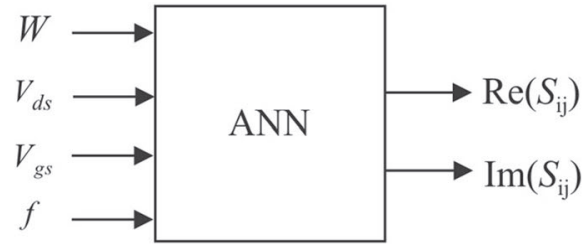

(B)

FIGURE 2 Neural model for modeling GaAs high-electron-mobility transistors with different gate width: (A) artificial neural network (ANN) exploited for modeling the imaginary and real parts separately (applied for $S_{21}$ and $S_{12}$ ) and (B) ANN exploited for simultaneously modeling the imaginary and real parts (applied for $S_{11}$ and $S_{22}$ )

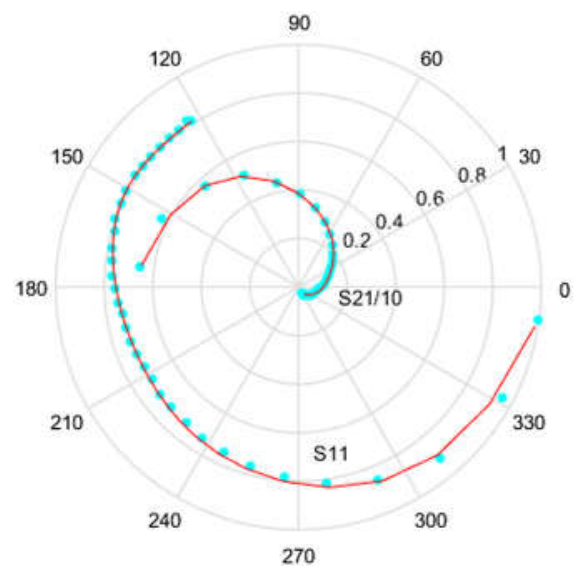

(A)

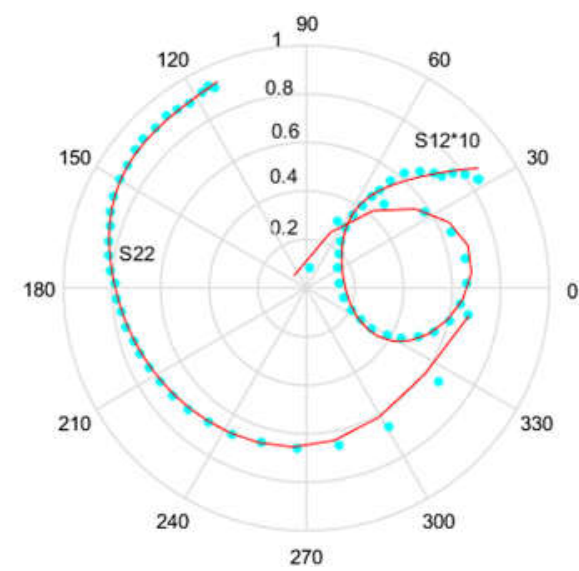

(B)

FIGURE 3 Comparison of the measured (symbols) and modeled (lines) $S$-parameters from 0.5 to $50 \mathrm{GHz}$ for the GaAs high-electronmobility transistor with a gate width of $300 \mu \mathrm{m}$ at $V_{\mathrm{ds}}=2.5 \mathrm{~V}$ and $V_{\mathrm{gs}}=-0.6 \mathrm{~V}$ : (A) $S_{11}$ and $S_{21}$ and (B) $S_{12}$ and $S_{22}$ 
by analyzing the errors, it was found that for the parameters having higher dynamics, high percentage errors can be achieved in the part of the frequency range where they exhibit very small values close to 0 . It is due to a relatively small absolute deviation that can make critical impact on the rise of the percentage errors. This issue was addressed in details in Marinković et al. ${ }^{43}$ It is worth mentioning that this problem is less critical when the parameters have small values in the whole frequency range, like in the case of $S_{12}$.

In addition, in Marinković et al, ${ }^{42,43}$ it was shown that the developed neural model provides better accuracy than a model based on the equivalent circuit (2\%-5\% better at least), except at the bias points with parameters exhibiting small values in a certain part of the frequency range.

In earlier work, the authors developed models for GaAs pseudomorpic HEMTs (pHEMTs) in a package for a limited range of biases (determined by the typical bias points given in the manufacturer's datasheets). The training data referred to two or three bias voltages. The models were developed for a single device ${ }^{41}$ and for three scaled devices differing in the gate width. ${ }^{40}$ In both cases, one ANN trained to model simultaneously all four $S$-parameters was enough to provide good modeling accuracy. The structure of the models is shown in Figure 4. For the single device (ie, ATF35143 by Hewlett Packard), the ANN has the following structure: 3-10-10-8, whereas for the scaled devices (ie, three pHEMTs: AT33143 with $W=400 \mu \mathrm{m}$, ATF34143 with $W=800 \mu \mathrm{m}$, and ATF35143 with $W=1600 \mu \mathrm{m}$ ), the ANN has the following structure: 3-7-7-8. It should be noted that the $S$-parameters were expressed in the magnitude-angle representation. To illustrate the achieved modeling accuracy, the $S$-parameters obtained by the developed neural models are shown and compared with the measured data in Figure 5. The plots refer to bias points not used for the model development. For the sake of better presentation, in Figure 5A, $S_{21}$ is divided by 8, and $S_{12}$ is multiplied by 5, while in Figure 5B, $S_{21}$ is divided by 10 and $S_{12}$ is multiplied by 3 . The modeling accuracy for the scaled model is slightly worse than in the case of the model for a single device, but the achieved results are still quite satisfactory. However, if needed, the accuracy can be improved by using previously mentioned models consisting of more ANNs.

It should be mentioned that if the $S$-parameters are given in the magnitude-angle representation, then a special attention should be paid to the range of expressing the angle of $S$-parameters $\left(0^{\circ}\right.$ to $360^{\circ}$ or $-180^{\circ}$ to $\left.180^{\circ}\right)$, as a sharp

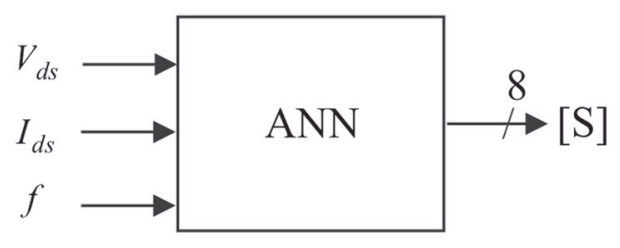

(A)

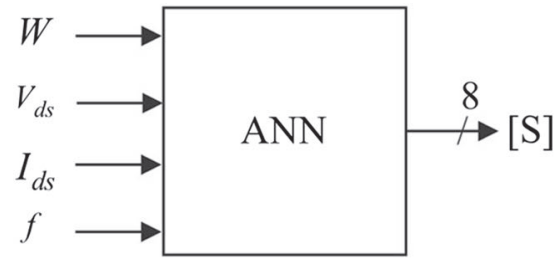

(B)

FIGURE 4 Neural model for modeling GaAs pseudomorpic high-electron-mobility transistor by exploiting a single artificial neural network (ANN) for the modeling of all four $S$-parameters: (A) one device model and (B) model for devices having different gate width

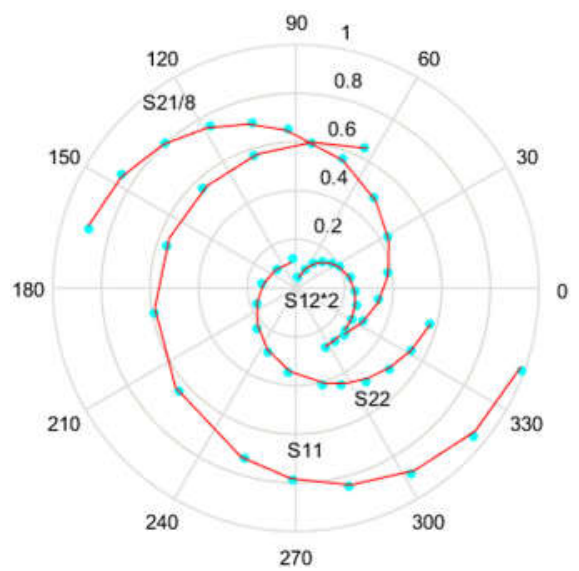

(A)

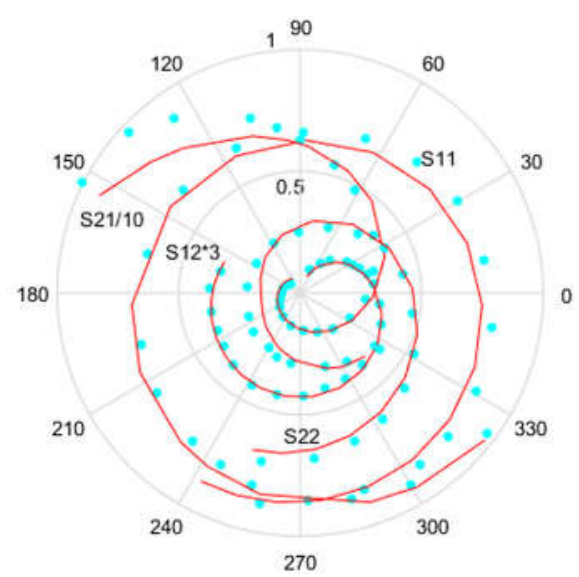

(B)

FIGURE 5 Comparison of the modeled (symbols) and simulated (lines) $S$-parameters of the GaAs pseudomorpic high-electron-mobility transistors: (A) single device model: ATF35143 at $V_{\mathrm{ds}}=2 \mathrm{~V}$ and $I_{\mathrm{ds}}=15 \mathrm{~mA}$ for frequencies up to $10 \mathrm{GHz}$ and (B) scalable model: ATF34143 at $V_{\mathrm{ds}}=3 \mathrm{~V}$ and $I_{\mathrm{ds}}=20 \mathrm{~mA}$ for frequencies up to $18 \mathrm{GHz}$ 
change that might appear in one of the chosen ranges would be very challenging to be modeled, resulting in significant deviations in the $S$-parameter modeling at the frequency range near this sharp change. ${ }^{41}$ This could be prevented by examining the behavior of the angles of the $S$-parameters and, then, choosing the angle range so that the sharp change does not appear in the angle-frequency dependence, as applied in the considered examples. However, to play safe, it is recommended to switch to the representation in the form of real and imaginary parts.

\section{2 | GaN HEMTs}

The neural approach was applied to an AlGaN/GaN HEMT on SiC substrate $\left(L_{\mathrm{g}}=0.7 \mu \mathrm{m}\right.$ and $\left.W=2 \times 400 \mu \mathrm{m}\right){ }^{47}$ The model was developed for the temperature range from $20^{\circ} \mathrm{C}$ to $80^{\circ} \mathrm{C}$ and frequency range from 0.3 to $40 \mathrm{GHz}$. The ranges of bias voltages were $V_{\mathrm{ds}}$ from 0 to $28 \mathrm{~V}$ and $V_{\mathrm{gs}}$ from -6 to $0 \mathrm{~V}$. A separate neural model was developed for each $S$ parameter consisting of two one-output ANNs aimed to model the real and imaginary part of the considered parameter. Therefore, these two ANNs have the structure shown in Figure 6A. However, to improve the modeling performance for $S_{21}$, its magnitude and angle were modeled instead of its real and imaginary parts. Moreover, because of a relatively high magnitude of $S_{21}$, the logarithmic representation was used for both $S_{21}$ magnitude and frequency (see Figure 6B) to make dynamics of the $S_{21}$ magnitude smaller and thus to improve ANN performance. Using the logarithmic representation of frequency also made the $S_{21}$ angle easier to be modeled.

The first models were developed with the training data uniformly sampled in the input space of biases and in the frequency range. However, as certain significant discrepancies appeared in the $S_{21}$ and $S_{12}$, a nonuniform sampling of bias points was performed taking more training data in the areas where the discrepancies appeared (ie, bias points referring to low $V_{d s}$ and in the transition region from the pinch-off to the maximum transconductance, as well as at low frequencies up to $5 \mathrm{GHz}$ ). The ANNs chosen for the final model were $S_{11}$ (Re: 4-24-22-1, Im: 4-25-25-1); $S_{21}$ (Log (Mag): 4-30-30-1, Ang: 4-28-28-1); $S_{12}$ (Re: 4-22-21-1, Im: 4-24-24-1); and $S_{22}$ (Re: 4-24-22-1, Im: 4-25-25-1). Analyzing the achieved modeling accuracy, it was found that the percentage errors averaged over the whole bias range for the temperatures used in the training set were lower than $3 \%$, and the maximum values were mostly lower than $6 \%$. As far as the temperature of $65^{\circ} \mathrm{C}$, which was not used for the model development is concerned, the errors were higher but at least lower than 5\%, except in the case of $S_{21}$. For this parameter, the higher error is attributed to the values close to 0 , where absolute differences between modeled and measured values are relatively small, as discussed in the previous section. Figure 7 illustrates a comparison of the modeled and measured values at $V_{\mathrm{ds}}=9.5 \mathrm{~V}$ and $V_{\mathrm{gs}}=-1.25 \mathrm{~V}$ at the ambient temperature of $65^{\circ} \mathrm{C}$. For the sake of a clearer plot, $S_{21}$ is divided by 20 , and $S_{12}$ is multiplied by 10 . The reported plot confirms that the developed model is capable to accurately reproduce the $S$-parameters, including the kink effect affecting $S_{22}$.

\section{3 | Si FinFET}

The Si FinFET device under investigation in Marinković et $\mathrm{a}^{44}$ was fabricated in IMEC technology. ${ }^{52}$ It has a gate length of $60 \mathrm{~nm}$ and a gate width of $27.36 \mu \mathrm{m}$. This device is a triple gate FinFET having a fin height of $60 \mathrm{~nm}$, a fin width of $32 \mathrm{~nm}$, and 30 fingers, where each finger is composed of six fins. The model was developed for the frequency range going from 0.3 to $50 \mathrm{GHz}$ over the range of biases with $V_{\mathrm{gs}}$ from 0 to $1.2 \mathrm{~V}$ and $V_{\mathrm{ds}}$ from 0 to $1.2 \mathrm{~V}$. Two cases were considered: modeling of the whole device and modeling of the actual device obtained by removing the parasitic effects of pads, transmission lines, and substrate by using an open and short de-embedding procedure. ${ }^{52}$ In both cases, separate

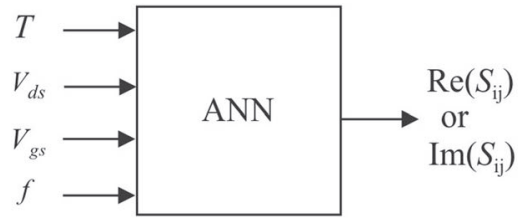

(A)

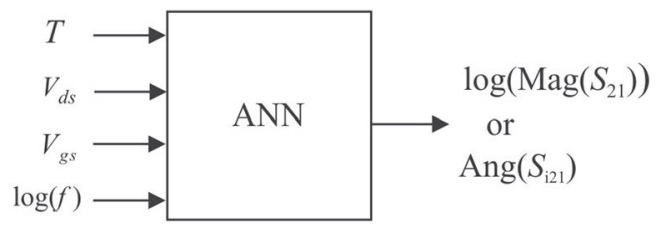

(B)

FIGURE 6 Neural model for temperature and bias dependent modeling of a GaN high-electron-mobility transistor: (A) artificial neural network (ANN) used for separate modeling of the real and imaginary parts of $S_{11}, S_{22}$, and $S_{12}$ and (B) ANN used for modeling of the $S_{21}$ magnitude and angle 


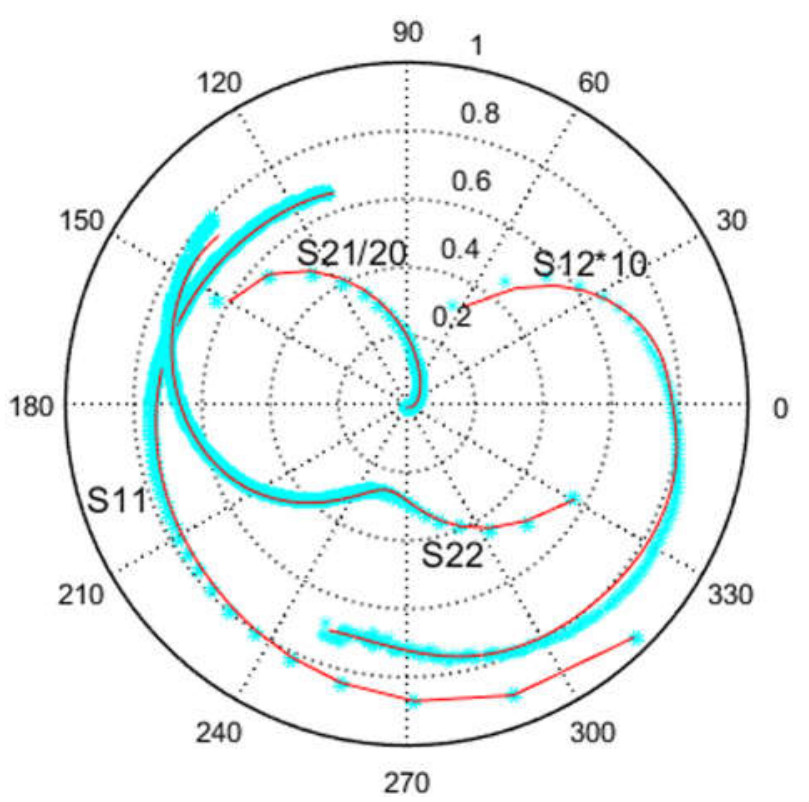

FIGURE 7 Comparison of the modeled (symbols) and simulated (lines) $S$-parameters from 0.3 to $40 \mathrm{GHz}$ for a GaN high-electron-mobility transistor at $V_{\mathrm{ds}}=9.5 \mathrm{~V}$ and $V_{\mathrm{gs}}=-1.25 \mathrm{~V}$ at the ambient temperature of $65^{\circ} \mathrm{C}$

neural models were developed for each of the four $S$-parameters. The actual device has a less complicated behavior, and the neural model for each parameter consists of a single ANN modeling simultaneously the real and imaginary parts of the modeled $S$-parameter (see Figure 8A) or two ANNs modeling separately the real and imaginary parts of the $S$ parameter (see Figure 8B). In the case of the whole device, a kink appears in the low-frequency behavior of all of the $S$-parameters, turning into a kink in the real part of the $S$-parameters. To ensure a good modeling accuracy, a special structure of the neural model was applied for the real part of the S-parameters. It is a two-step model based on the so-called PKI principle. Namely, the real part neural model consists of two ANNs, as shown in Figure 8C. The first one is trained to model the real part in the low-frequency range where the kink occurs (in the particular case up to

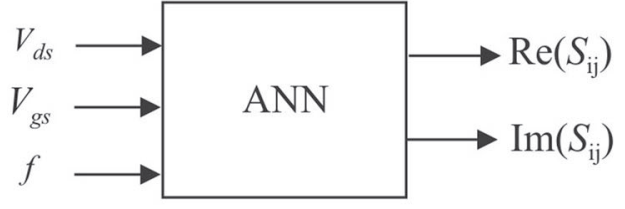

(A)

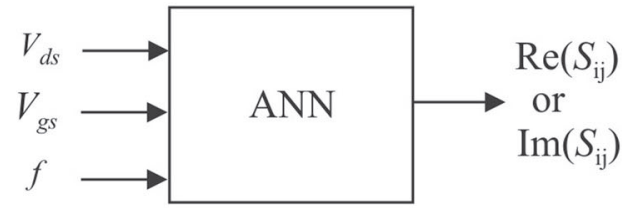

(B)

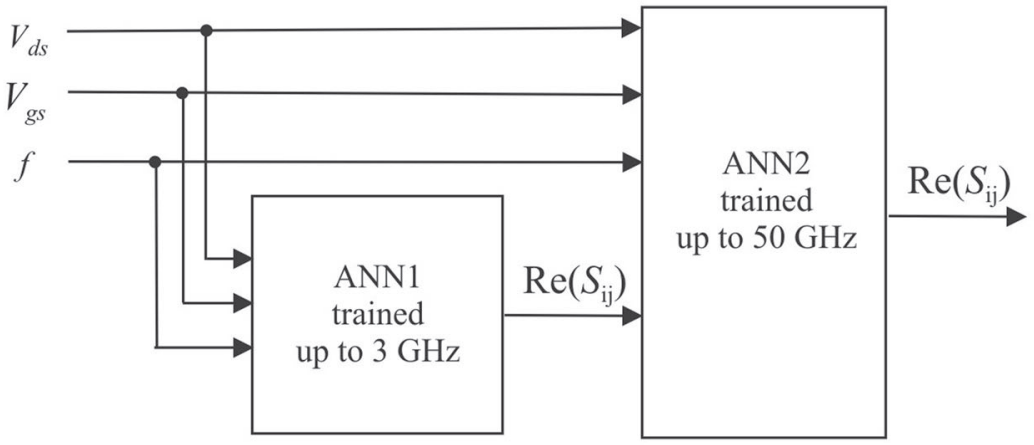

(C)

FIGURE 8 Neural model for bias dependent modeling of an Si fin field-effect transistor: (A) artificial neural network (ANN) used for simultaneous modeling of the real and imaginary parts of $S_{11}, S_{22}$, and $S_{12}$ of the actual device; (B) ANN used for separate modeling of the real and imaginary parts of $S_{21}$ of the actual device and of the imaginary parts of all $S$-parameters of the whole device; and (C) two-step ANN model used for modeling of the real parts of all $S$-parameters of the whole device 
$3 \mathrm{GHz}$ ). The second ANN is trained to model the real part in the whole frequency range up to $50 \mathrm{GHz}$. This ANN has the output of the ANN1 as an additional input besides the bias voltages and frequency. For modeling the $S$-parameter imaginary part, the one-outputANN as shown in Figure 8B was used.

The measured data available for the model development were referred to 325 bias points; out of them, 91 were used for the ANN training, and the other bias points were used for the model validation.

The developed model for the actual device consisted of three ANNs modeling simultaneously the real and imaginary parts of the parameters $S_{11}$ (3-20-20-2), $S_{12}$ (3-24-22-2), and $S_{22}$ (3-30-30-2), and two ANNs aimed at modeling the real and imaginary parts of $S_{21}$ (Re: 3-27-23-1, Im:3-26-25-1). The obtained model provided accuracy around 3\% (averaged over the bias range). For illustration, Figure 9A shows the simulated $S$-parameters compared with the measured data for the bias point $V_{\mathrm{ds}}=0.7 \mathrm{~V}$ and $V_{\mathrm{gs}}=0.45 \mathrm{~V}$. To make the plots clearer, $S_{12}$ is multiplied by 2 , and $S_{11}$ is translated by -0.2 .

In the case of the $S$-parameters for the whole device, the real parts were modeled by using two-step model, and the imaginary parts were modeled by using a single-output ANN. The following ANNs composed the final model: $\mathrm{S}_{11}$ (Re: ANN1 3-22-20-1, ANN2 4-25-25-1, Im: 3-20-20-1), S 21 (Re: ANN1 3-15-15-1, ANN2 4-25-24-1, Im: 3-24-22-1), S ${ }_{12}$ (Re: ANN1 3-25-25-1, ANN2 4-25-25-1, Im: 3-25-25-1), and $S_{22}$ (Re: ANN1 3-12-12-1, ANN2 4-24-20-1, Im: 3-25-25-1). The percentage error averaged over the bias range (for all 325 bias points) is less than $3 \%$ in the case of all four $S$-parameters, whereas the maximum error per bias point is less than 5\%. It is important that this model accurately reproduces also the low-frequency kinks, as illustrated in Figure 9B, which refer to the bias point $V_{\mathrm{ds}}=0.7 \mathrm{~V}$ and $V_{\mathrm{gs}}=0.45 \mathrm{~V}$. To have the plots clearer, $S_{12}$ is multiplied by 4 , and $S_{11}$ and $S_{22}$ are translated by -0.1 and +0.1 , respectively. More details about the model development and validation, especially details about the kink modeling, can be found in Marinković et al. ${ }^{47} \mathrm{~A}$ similar approach was also applied to modeling of the FinFET admittance parameters, resulting in models very convenient to be further applied in large-signal modeling. ${ }^{45}$

\section{4 $\mid$ Si FinFET varactors}

The neural modeling approach was applied to a varactor fabricated in FinFET technology on silicon-on-insulator (SOI) substrate, which is an nMOS transistor with common source and drain. ${ }^{52,55}$ It consists of eight devices connected in parallel, and each device is made of 16 gate fingers covering five fins. The geometrical dimensions are a fin height of $60 \mathrm{~nm}$, a fin width of $0.67 \mu \mathrm{m}$, and a gate length of $0.385 \mu \mathrm{m}$. The model was developed over the range of bias voltage $V_{\text {dio }}$ representing the voltage difference between anode (ie, gate) and ground, while the cathode (ie, source and drain) is fixed at the ground potential. ${ }^{52,55}$ The range of the considered $V_{\text {dio }}$ values was from -0.5 to $1.5 \mathrm{~V}$. The frequency range was from 45 to $50 \mathrm{GHz}$. The model was developed for the whole device and for the actual device obtained applying the deembedding procedure based on an open dummy structure. ${ }^{52,55}$ The ANN structure used for modeling the $S$-parameters is an ANN having the voltage $V_{\text {dio }}$ and the frequency as the inputs and the real and imaginary parts as the outputs, as shown in Figure 10.

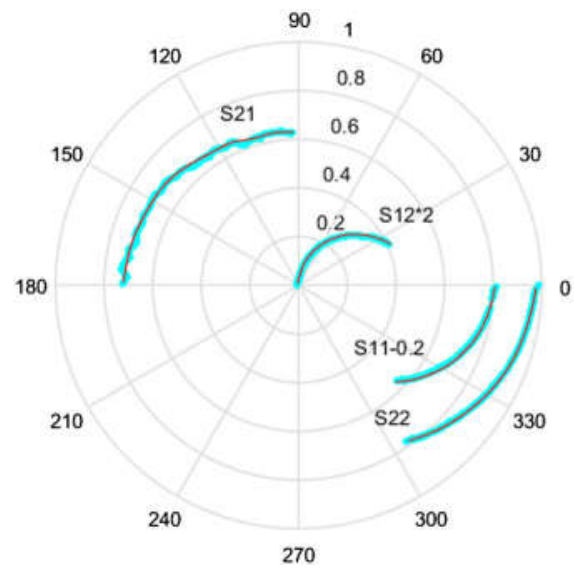

(A)

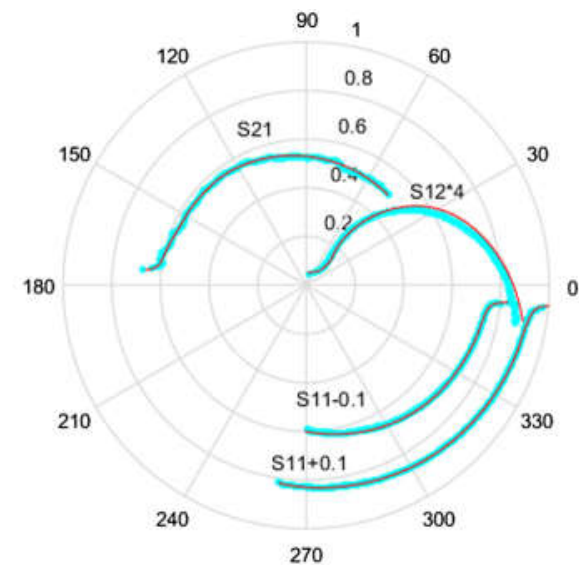

(B)

FIGURE 9 Comparison of the modeled (symbols) and simulated (lines) $S$-parameters of the fin field-effect transistor at $V_{\mathrm{ds}}=0.7 \mathrm{~V}$ and $V_{\mathrm{gs}}=0.45 \mathrm{~V}$ : (A) actual device and (B) whole device 


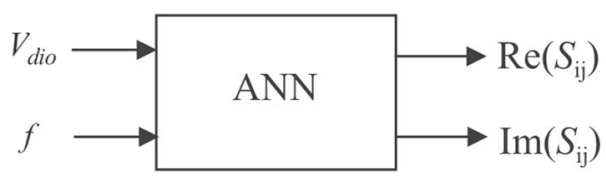

FIGURE 10 Neural model for bias dependent modeling of a Si fin field-effect transistor varactor: artificial neural network (ANN) used for simultaneously modeling the real and imaginary parts of each $S$-parameter

In the case of the whole device, the varactor behaves as a symmetric and reciprocal network; therefore, it follows that $S_{11}=S_{22}$ and $S_{12}=S_{21}$, implying that it is enough to develop models for $S_{11}$ and $S_{21}$. The ANNs composing the final model had the same structure with two hidden layers containing 15 neurons each (2-15-15-2). The achieved percentage errors averaged over the considered bias range are smaller than $2 \%$, confirming a good modeling accuracy. Although it is supposed that $S_{11}=S_{22}$ and $S_{12}=S_{21}$, the corresponding percentage errors were not equal, because the measured data are not identical, due to the measurement uncertainty and/or inevitable asymmetries in the device layout/structure. Figure 11 illustrates the comparison of the simulated and measured $S$-parameters from 45 to $50 \mathrm{GHz}$ for the bias point $V_{\text {dio }}=0.2 \mathrm{~V}$ that was not used for the model development.

In the case of the actual device, it behaves like a simple series impedance between anode and cathode implying that $S_{11}=S_{22}=1-S_{12}=1-S_{21}{ }^{55}$ Therefore, it is enough to develop a model for $S_{11}$ and calculate the other three parameters. Based on the available data, the best trained ANN was 2-16-15-2. The averaged percentage error for $S_{11}$ was $2.2 \%$. However, as the $S_{11}$ modeling error propagates to the other three parameters, their errors had different values. The measurement uncertainty also contributes to the difference in the errors. The error propagation affects particularly the $S_{22}$ parameter in the frequency range where it exhibits small values close to 0 (which is not the case for the whole device) resulting in significant percentage errors (20\%-40\%). For this reason, it is recommended to develop a model for $S_{11}$, to calculate $S_{12}$ and $S_{21}$ and develop a separate model for $S_{22}$, as it was done in the considered case. The best ANN for $S_{22}$ had the structure 2-16-15-2, providing the accuracy similar to the other $S$-parameters. To illustrate the modeling accuracy, the $S$-parameters from 45 to $50 \mathrm{GHz}$ were obtained by the neural model and compared with the measured data for the bias point not used in the model development (see in Figure 12).

\section{5 | Si MOSFETs}

The ANNs were applied to model three multicelled multi-finger Si MOSFETs with a gate width of $192 \mu \mathrm{m}$ $(W=16 \times 4 \times 3,8 \times 4 \times 6$, and $8 \times 4 \times 6 \mu \mathrm{m})$ and different gate lengths $(L=0.25,0.5$, and $1 \mu \mathrm{m})$. The model was developed for the frequency range going from 0.3 to $40 \mathrm{GHz}$ and for the following bias range: $V_{\mathrm{gs}}$ from 0 to $1 \mathrm{~V}$ and $V_{\mathrm{ds}}$ from 0 to $1 \mathrm{~V}^{49}$ The neural model for each $S$-parameter was developed separately. It was found that the best results were obtained when the real and imaginary parts of a modeled parameter were modeled by two separate ANNs, having

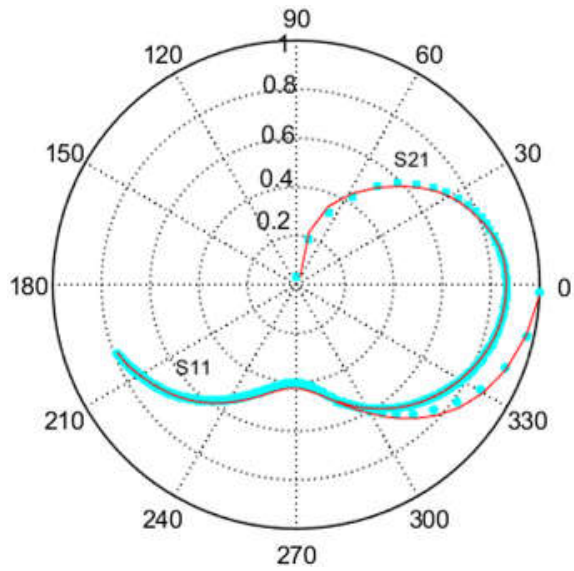

(A)

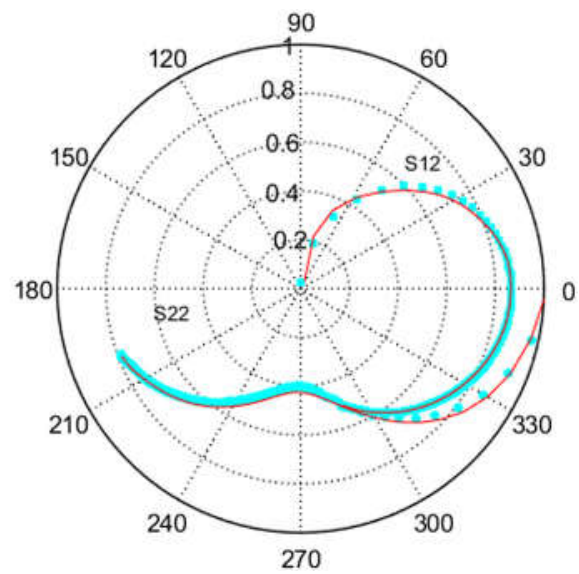

(B)

FIGURE 11 Comparison of the measured (symbols) and simulated (lines) $S$-parameters from 45 to $50 \mathrm{GHz}$ for the whole Si fin field-effect transistor varactor at $V_{\text {dio }}=0.2 \mathrm{~V}$ : (A) $S_{11}$ and $S_{21}$ and (B) $S_{12}$ and $S_{22}$ 


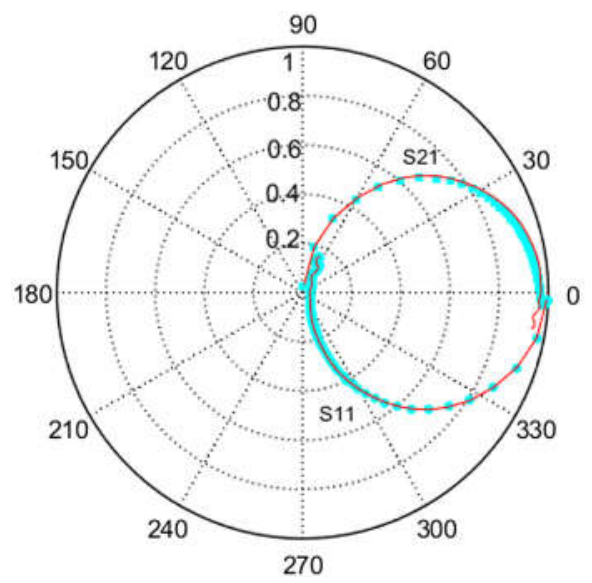

(A)

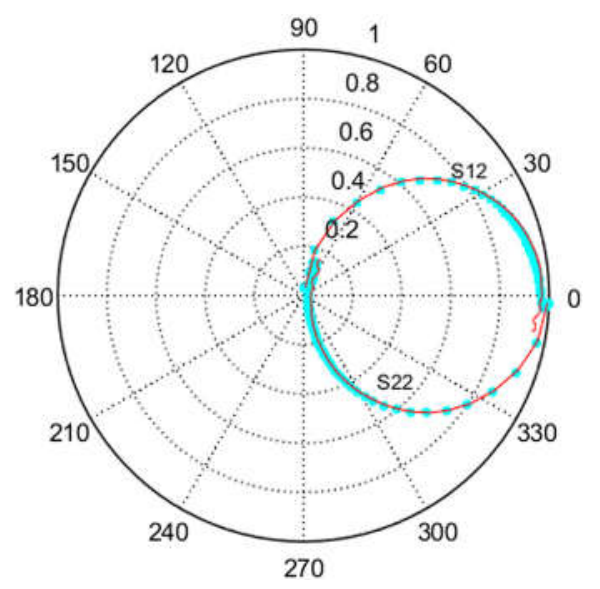

(B)

FIGURE 12 Comparison of the measured (symbols) and simulated (lines) $S$-parameters from 45 to $50 \mathrm{GHz}$ for the actual Si fin field-effect transistor varactor at $V_{\text {dio }}=0.2 \mathrm{~V}$ : (A) $S_{11}$ and $S_{21}$ and (B) $S_{12}$ and $S_{22}$

the structure shown in Figure 13. The ANNs have four inputs corresponding to the gate length, two bias voltages, and frequency. The ANNs have only one output corresponding to the real or imaginary part of the modeled $S$-parameter.

The developed neural model consists of the following ANNs: $S_{11}$ (Re: 4-10-10-1, Im: 4-12-12-1), $S_{21}$ (Re: 4-12-12-1, Im: 414-14-1), $S_{12}$ (Re: 4-10-10-1, Im: 4-10-10-1), and $S_{22}$ (Re: 4-10-10-1, Im: 4-10-10-1). Although the available set of measured data was relatively small (it referred to only 30 bias points) and only a part of it was used for the model development, the

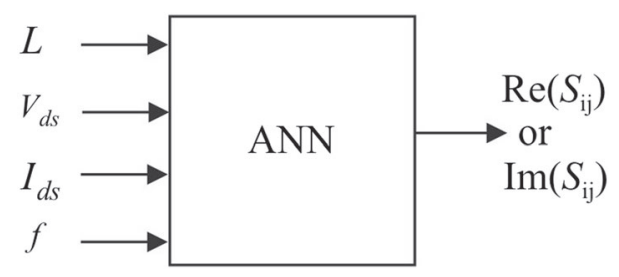

FIGURE 13 Neural model for bias dependent modeling of Si metal-oxide-semiconductor field-effect transistors with different gate length: artificial neural network (ANN) used for modeling of the real and imaginary part of each $S$-parameter

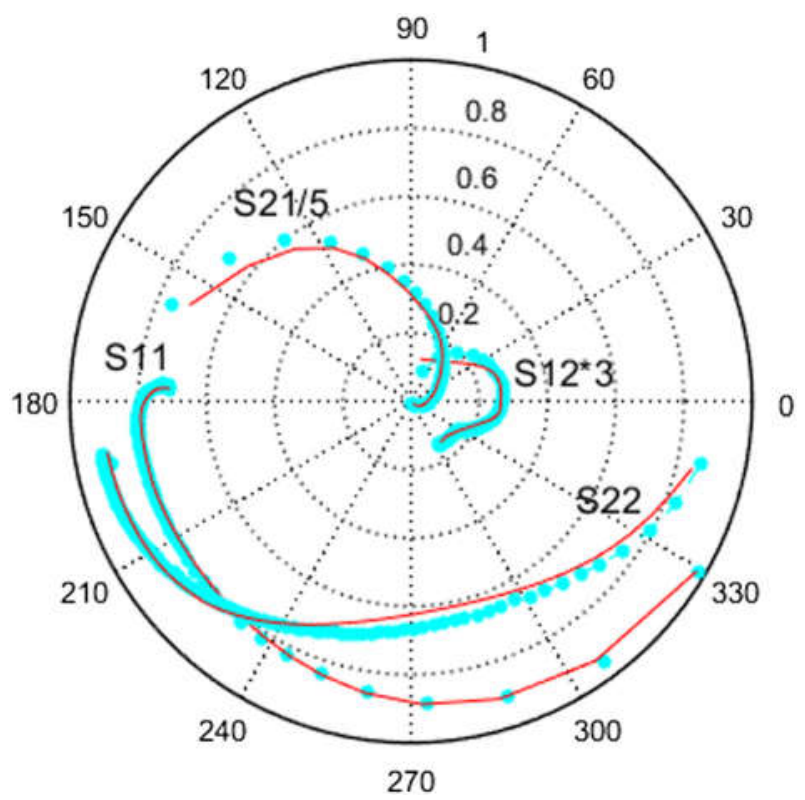

FIGURE 14 Comparison of the measured (symbols) and simulated (lines) S-parameters from 0.3 to $40 \mathrm{GHz}$ for a Si metal-oxidesemiconductor field-effect transistor with a gate length of $500 \mathrm{~nm}$ at $V_{\mathrm{gs}}=0.8 \mathrm{~V}$ and $V_{\mathrm{ds}}=0.75 \mathrm{~V}$ 
achieved modeling errors were very acceptable. Namely, for all of the three devices, the percentage error averaged over the bias and frequency range was less than $1 \%$, except for the parameter $S_{21}$ where the error was less than $2.6 \%$. However, that higher error can be attributed to the small deviations of the values very close to 0 resulting in high percentage errors. As a proof of a good modeling accuracy, Figure 14 reports the plots of the $S$-parameters obtained by the model and the measured values for the bias point with $V_{\mathrm{gs}}=0.8 \mathrm{~V}$ and $V_{\mathrm{ds}}=0.75 \mathrm{~V}$, not used for the model development, for the device having a gate length of $500 \mathrm{~nm}$. For a clearer plot, $S_{21}$ is divided by 5 , and $S_{12}$ is multiplied by 3 .

\section{4 | CONCLUSIONS}

The ANNs can be successfully applied to model the small-signal scattering parameters of microwave FETs made in different technologies. We have developed models for several technologies and compared them in this paper. The developed models are bias and frequency dependent, and in certain cases, the dependence on the ambient temperature or on the device dimensions is considered. By analyzing and comparing the achieved experimental finding, it can be concluded that if the considered bias range is relatively narrow (ie, referring only to the bias points for the recommended applications of the transistor), then all of the $S$-parameters can be modeled by a single ANN. This can be explained by the fact that at the typical bias points, the $S$-parameters exhibit roughly a similar behavior. In such cases, it is enough to use a single ANN for all the $S$-parameters also for scalable models.

By selecting a broad bias range in order to cover all of the device working regimes, including the bias points where the $S$-parameter behavior is different than for the typical bias points (such as the "pinch-off" condition), it is recommended to develop separate models for each of the $S$-parameters. Depending on the parameter behavior, the real and imaginary parts are modeled simultaneously by a single ANN or separately by two different ANNs. However, in certain cases, even having the real and imaginary parts modeled by separate ANNs, it is not enough for a satisfactory accuracy. In that critical case, it is necessary to analyze the modeling errors for identifying and understanding the reason of their increase. For instance, a nonuniform sampling of the training data, having more samples in the regions where the errors are higher, can help to make the errors smaller. If even this nonuniform sampling leads to errors that are still not acceptable, then more complex model structures should be introduced, as in the shown case of Si FinFETs having low-frequency kink effects. In this case, a separate model was trained for the low-frequency range where the kink effects appear, and then, it was integrated in the final model covering the whole frequency range. Sometimes, different representations of the parameters to be modeled should be exploited, in order to make their dependence easier to be modeled, as in the case of the GaN HEMTs having $S_{21}$ with a high dynamic. In this case, it is more convenient to use magnitude and angle representation for $S_{21}$ instead of the representation in terms of the real and imaginary parts. Moreover, $S_{21}$ magnitude and the frequency are expressed in logarithmic scale to decrease the parameter dynamics and make its modeling easier and, consequently, the error smaller.

When estimating the modeling errors, one should be aware that for the parameters having values close to 0 , small absolute differences may produce very high percentage errors. This usually might happen for $S_{12}$, which typically exhibits small values and can get quite critical for $S_{21}$, which has a higher dynamics, and then the small absolute difference does not contribute significantly in the overall ANN training performance. Therefore, the percentage errors should not be taken as an absolute indicator of the model accuracy. Comparisons of the correlation coefficients between the simulated and reference values and a visual inspection of the modeled characteristics can be used as additional tools to establish the model accuracy.

Finally, it can be concluded that the observed differences in the structure of the neural models depend strongly on the device technology, which influences the device behavior and the "shape" of the characteristics to be modeled. However, despite the exhibited differences, all of the developed ANN-based models provide very good modeling accuracy and, therefore, are suitable to be used for further development of accurate large-signal and noise models of the studied devices.

\section{ACKNOWLEDGEMENT}

This work was supported by the Serbian Ministry for Education and Science within the project TR-32052.

\section{ORCID}

Zlatica Marinković (10) https://orcid.org/0000-0002-2954-7275

Giovanni Crupi (D) https://orcid.org/0000-0002-6666-6812 


\section{REFERENCES}

1. Zhang QJ, Gupta KC. Neural Networks for RF and Microwave Design. Artech House: Boston, MA; 2000.

2. Burrascano P, Fiori S, Mongiardo M. A review of artificial neural network applications in microwave computer-aided design. Int $J$ RF Microw Comput-Aided Eng. 1999;9(3):158-174.

3. Wang F, Devabhaktuni VK, Xi C, Zhang QJ. Neural network structures and training for RF and microwave applications. Int $J$ RF and Microw Comput-Aided Eng. 1999;9(3):216-240.

4. Bandler JW, Ismail MA, Rayas-Sánchez JE, Zhang QJ. Neuromodeling of microwave circuits exploiting space mapping technology. IEEE Trans Microw Theory Techn. 1999;47(2):2417-2427.

5. Patnaik A, Mishra RK. ANN techniques in microwave engineering. IEEE Microw Mag. 2000;1(1):55-60.

6. Devabhaktuni VK, Yagoub MCE, Fang Y, Xu JJ, Zhang QJ. Neural networks for microwave modeling: model development issues and nonlinear modeling techniques. Int J RF and Microw Comput-Aided Eng. 2001;11(1):4-21.

7. Giannini F, Leuzzi G, Orengo G, Albertini M. Small-signal and large-signal modeling of active devices using CAD-optimized neural networks. Int J RF Microw Comput-Aided Eng. 2002;12(1):71-78.

8. Zhang QJ, Gupta KC, Devabhaktuni VK. Artificial neural networks for RF and microwave design—from theory to practice. IEEE Trans Microw Theory Techn. 2003;51(4):1339-1350.

9. Schreurs D, Verspecht J, Vandamme E, Vellas N, Gaquiere C, Germain M. ANN model for AlGaN/GaN HEMTs constructed from nearoptimal-load large-signal measurements. IEEE Int Microw Symp. 2003;1:447-450.

10. Rayas-Sánchez JE. EM-based optimization of microwave circuits using artificial neural networks: the state of the art. IEEE Trans Microwave Theory Techn. 2004;52(1):420-435.

11. Marinković Z, Marković V. Temperature dependent models of low-noise microwave transistors based on neural networks. Int $J$ RF Microw Comput-Aided Eng. 2005;15(6):567-577.

12. Rayas-Sánchez JE, Gutiérrez-Ayala V. EM-based Monte Carlo analysis and yield prediction of microwave circuits using linear-input neural-output space mapping. IEEE Trans Microwave Theory Techn. 2006;54(12):4528-4537.

13. Caddemi A, Catalfamo F, Donato N. A neural network approach for compact cryogenic modeling of HEMT's. Int J Electron. 2007;94(9):877-887.

14. Koziel S, Bandler JW. Modeling of microwave devices with space mapping and radial basis functions. Int J Numer Model Electron Network Dev Field. 2007;21(3):187-203.

15. Simsek M, Sengor NS. A knowledge-based neuromodeling using space mapping technique: compound space mapping-based neuromodeling. Int J Numer Model Electron Network Dev Field. 2008;21(1/2):133-149.

16. Zhang L, Zhang QJ. Neuro-space mapping technique for semiconductor device modeling. Optim Eng. 2008;9(4):393-405.

17. Cao Y, Zhang QJ. A new training approach for robust recurrent neural-network modeling of nonlinear circuits. IEEE Trans Microwave Theory Tech. 2009;57(6):1539-1553.

18. Kabir H, Zhang L, Yu M, Aaen P, Wood J, Zhang QJ. Smart modeling of microwave devices. IEEE Microw Mag. 2010;11(3):105-108.

19. Gutiérrez-Ayala V, Rayas-Sánchez JE. Neural input space mapping optimization based on nonlinear two-layer perceptrons with optimized nonlinearity. Int J RF and Microw Comput-Aided Eng. 2010;20(5):512-526.

20. Kabir H, Yu M, Zhang QJ. Recent advances of neural network-based EM-CAD. Int J RF and Microw Comput-Aided Eng. 2010;20(5):502-511.

21. Cao Y, Erdin I, Reitzinger S, Zhang QJ. Simple and efficient high-dimensional parametric modeling for microwave cavity filters using modular neural network. IEEE Microw Wireless Comp Lett. 2011;21(5):258-260.

22. Murphy EK, Yakovlev VV. Neural network optimization of complex microwave structures with a reduced number of full-wave analyses. Int J RF and Microw Comput-Aided Eng. 2011;21(3):279-287.

23. Barmuta P, Plonski P, Czuba K, Avolio G, Schreurs D. Nonlinear AlGaN/GaN HEMT model using multiple artificial neural networks. Proc. of 19th International Conference on Microwave Radar and Wireless Communications - MIKON 2012, Warsaw, Poland, May 2012; 462-466.

24. Marinković Z, Ivković N, Pronić-Rančić O, Marković V, Caddemi A. Analysis and validation of neural approach for extraction of smallsignal models of microwave transistors. Microelectron Reliab. 2013;53(3):414-419.

25. Hayatia M, Akhlaghi B. An extraction technique for small signal intrinsic parameters of HEMTs based on artificial neural networks. $A E U$ - Int J Electron Comm. 2013;67(2):123-129.

26. Cao Y, Wang G, Gunupudi P, Zhang QJ. Parametric modeling of microwave passive components using combined neural networks and transfer functions in the time and frequency. Int J RF and Microw Comput-Aided Eng. 2013;23(1):20-33.

27. Jarndal J. Genetic algorithm-based neural-network modeling approach applied to AlGaN/GaN devices. Int J RF Microwave Comput-Aided Eng. 2013;23(2):507-516.

28. Crupi G, Raffo A, Marinković $\mathrm{Z}$, et al. An extensive experimental analysis of the kink effects in $\mathrm{S}_{22}$ and $\mathrm{h}_{21}$ for a GaN HEMT. IEEE Trans Microwave Theory Techn. 2014;62(3):513-520. 
29. Na WC, Zhang QJ. Automated knowledge-based neural network modeling for microwave applications. IEEE Microw Wirel Compon Lett. 2014;24(7):499-501.

30. Aguilar-Lobo LM, Loo-Yau JE, Rayas-Sánchez JE, Ortega-Cisneros S, Moreno P, Reynoso-Hernández JA. Application of the NARX neural network as a digital predistortion technique for linearizing microwave power amplifiers. Microw Opt Techn Lett. 2015;57(9):2137-2142.

31. Khan T, De A. Modeling of microstrip antennas using neural networks techniques: a review. Int J RF Microw Comput-Aided Eng. 2015;25(9):747-757.

32. Feng F, Zhang C, Ma J, Zhang QJ. Parametric modeling of EM behavior of microwave components using combined neural networks and pole-residue-based transfer functions. IEEE Trans Microw Theory Techn. 2016;64(1):60-77.

33. Marinković Z, Marković V, Ćirić T, Vietzorreck L, Pronić-Rančić O, Marković V. Artifical neural networks in RF MEMS switch modeling. Facta Universitatis, Series: Electronics and Energetics. 2016;29(2):177-191.

34. Zhu L, Liu K, Liu W, et al. An advanced analytical neuro-space mapping technique with sensitivity analysis for transistor modeling. Int $J$ Numer Model Electron Network Dev Field. 2016;30(6):e2206.

35. Aoad A, Simsek M, Aydin Z. Knowledge-based response correction method for design of reconfigurable N-shaped microstrip patch antenna using inverse ANNs. Int J Numer Model Electron Network Dev Field. 2017;30(3-4):e2129.

36. Gunes F, Mahouti P, Demirel S, Belen M, Uluslu A. Cost-effective GRNN-based modeling of microwave transistors with a reduced number of measurements. Int J Numer Model Electron Network Dev Field. 2017;30(3-4):e2089.

37. Zhu YY, Ma JG, Fu HP, Zhang QJ, Cheng QF, Lin Q. Accurate modeling of pHEMT output current derivatives over a wide temperature range. Int J Numer Model Electron Network Dev Field. 2017;30(3-4):e2185.

38. Ćirić T, Dhuri R, Marinković Z, Pronić-Rančić O, Marković V, Vietzorreck L. Neural based lumped element model of capacitive RF MEMS switches. Frequenz. 2018;72(11-12):539-546.

39. Viveros-Wacher A, Rayas-Sanchez JE. Analog fault identification in RF circuits using artificial neural networks and constrained parameter extraction. Proc. of the IEEE MTT-S International Conference on Numerical Electromagnetic and Multiphysics Modeling and Optimization (NEMO 2018), Reykjavik, Iceland, August 2018; 1

40. Marinković Z, Pronić O, Marković V. Bias-dependent scalable modeling of microwave FETs based on artificial neural networks. Microw Opt Techn Lett. 2006;48(10):1932-1936.

41. Marinković Z, Stošić A, Marković V, Pronić O. ANNs in bias-dependent modeling of S-parameters of microwave FETs and HBTs. Microwave Review. 2006;1(12):21-30.

42. Marinković Z, Crupi G, Caddemi A, Marković V. Comparison between analytical and neural approaches for multi-bias small signal modeling of microwave scaled FETs. Microw Opt Techn Lett. 2010;52(10):2238-2244.

43. Marinković Z, Crupi G, Caddemi A, Marković V. On the neural approach for FET small-signal modelling up to 50GHz. 10th Seminar of Neural Network Application in Electrical Engineering - NEUREL 2010, Belgrade, Serbia, September 2010; 89-92.

44. Marinković Z, Crupi G, Schreurs D, Caddemi A, Marković V. Microwave FinFET modeling based on artificial neural networks including lossy silicon substrate. Microelectron Eng. 2011;88(10):3158-3163.

45. Marinković Z, Crupi G, Schreurs D, Caddemi A, Marković V. Multi-bias neural modeling of FinFET admittance parameters. Microw Opt Techn Lett. 2012;54(9):2082-2088.

46. Marinković Z, Crupi G, Schreurs D, Caddemi A, Marković V. Microwave neural modeling for silicon FinFET varactor. Int J Numer Model Electron Network Dev Field Electron Networks Devices Fields. 2014;27(5-6):834-845.

47. Marinković Z, Crupi G, Caddemi A, et al. Neural approach for temperature dependent modeling of GaN HEMTs. Int J Numer Model Electron Network Dev Field. 2015;28(4):359-370.

48. Marinković Z, Crupi G, Schreurs D, Caddemi A, Marković V. GaN HEMT small-signal modelling: neural networks versus equivalent circuit. Proc. 23th Int Conf Microelectronics (MIEL 2017), Niš, Serbia, October 2017;153-156.

49. Marinković Z, Crupi G, Schreurs D, Caddemi A, Marković V. Neural procedure for microwave MOSFET modelling versus bias and gate length. Proc of 13th Int Conf Advanced Techn Systems and Services in Telecom (TELSIKS 2017), Niš, Serbia, October 2017;166-169.

50. Dambrine G, Cappy A, Heliodore F, Playez E. A new method for determining the FET small-signal equivalent circuit. IEEE Trans Microwave Theory Techn. 1988;36(7):1151-1159.

51. Crupi G, Xiao D, Schreurs DMM-P, et al. Accurate multibias equivalent circuit extraction for GaN HEMTs. IEEE Trans Microwave Theory Techn. 2006;54(10):3616-3622.

52. Crupi G, Schreurs DMM-P, Caddemi A, et al. Purely analytical extraction of an improved nonlinear FinFET model including non-quasistatic effects. Microelectron Eng. 2009;86(11):2283-2289.

53. Crupi G, Schreurs DMM-P, Caddemi A. On the small signal modeling of advanced microwave FETs: a comparative study. Int J RF Microwave Comput-Aided Eng. 2008;18(5):417-425.

54. Xu Y, Guo Y, Xu R, Yan B, Wu Y. An improved small-signal equivalent circuit model for 4H-SiC power MESFETs. Microwave Opt Techn Lett. 2008;50(6):1455-1458.

55. Crupi G, Schreurs DMM-P, Dehan M, et al. Analytical extraction of small and large signal models for FinFET varactors. Solid State Electron. 2008;52(5):704-710. 
56. Choi W, Jung G, Kim J, Kwon Y. Scalable small-signal modeling of RF CMOS FET based on 3-D EM-based extraction of parasitic effects and its application to millimeter-wave amplifier design. IEEE Trans Microwave Theory Techn. 2009;57(12):3345-3353.

57. Resca D, Raffo A, Santarelli A, Vannini G, Filicori F. Scalable equivalent circuit FET model for MMIC design identified through FW-EM analyses. IEEE Trans Microwave Theory Techn. 2009;59(2):245-253.

58. Crupi G, Schreurs DMMP, Raskin JP, Caddemi A. A comprehensive review on microwave FinFET modeling for progressing beyond the state of art. Solid State Electron. 2013;80:81-95.

59. Jarndal A. AlGaN/GaN HEMTs on $\mathrm{SiC}$ and $\mathrm{Si}$ substrates: a review from the small-signal-modeling's perspective. Int $J$ RF Microwave Comput-Aided Eng. 2014;24(3):389-400.

60. Shen L, Chen B, Gao J. An improved millimeter-wave small-signal modeling approach for HEMTs. Int J RF Microwave Comput-Aided Eng. 2014;24(4):464-469.

61. Bosi G, Crupi G, Vadala V, Raffo A, Giovannelli A, Vannini G. Nonlinear modeling of LDMOS transistors for high-power FM transmitters. Int J Numer Model Electron Network Dev Field. 2014;27(5-6):780-791.

62. Nalli A, Raffo A, Crupi G, et al. GaN HEMT noise model based on electromagnetic simulations. IEEE Trans Microw Theory Techn. 2015;63(8):2498-2508.

63. Karisan Y, Caglayan C, Trichopoulos GC, Sertel K. Lumped-element equivalent-circuit modeling of millimeter-wave HEMT parasitics through full-wave electromagnetic analysis. IEEE Trans Microwave Theory Techn. 2016;64(5):1419-1430.

64. Crupi G, Caddemi A, Schreurs DMMP, Dambrine G. The large world of FET small-signal equivalent circuits. Int J RF Microwave ComputAided Eng. 2016;26(9):749-762.

65. Jarndal A, Kouki A. Efficient modeling of GaN HEMTs for linear and nonlinear circuits design. Int J Numer Model Electron Network Dev Field. 2017;30(1):e2100.

66. Wen Z, Xu Y, Wang C, Zhao X, Xu R. An efficient parameter extraction method for GaN HEMT small-signal equivalent circuit model. Int J Numer Model Electron Network Dev Field. 2017;30(1):e2127.

67. Chen Y, Xu Y, Wang F, et al. A scalable and multibias parameter extraction method for a small-signal GaN HEMT model. Int J Numer Model Electron Network Dev Field. 2017;31(5):e2347.

68. Hussein AS, Jarndal AH. Reliable hybrid small-signal modeling of GaN hemts based on particle-swarm-optimization. IEEE Trans Comput-Aided Design Integr Circuits Syst. 2018;37(9):1816-1824.

69. Snowden CM. Microwave and millimeter-wave device and circuit design based on physical modeling. Int J RF Microwave Comput-Aided Eng. 1991;1(1):4-21.

70. Guerrieri SD, Bonani F, Pirola M, Ghione G. Sensitivity-based optimization and statistical analysis of microwave semiconductor devices through multidimensional physical simulation. Int J RF Microwave Comput-Aided Eng. 1997;7(1):129-143.

71. Bonani F, Guerrierim SD, Ghione G. Physics-based simulation techniques for small- and large-signal device noise analysis in RF applications. IEEE Trans Electron Devices. 2003;50(3):633-644.

72. Denis D, Snowden CM, Hunter IC. Coupled electrothermal, electromagnetic, and physical modeling of microwave power FETs. IEEE Trans Microwave Theory Techn. 2006;54(6):2465-2470.

73. Sippel JC, Islam SS, Mukherjee SS. A physics-based model of DC and microwave characteristics of GaN/AlGaN HEMTs. Int J RF Microwave Comput-Aided Eng. 2007;17(3):254-264. 